\title{
RNA expression of the molecular signature genes for metastasis in colorectal cancer
}

\author{
LUCIANO CARVALHO ${ }^{1-3^{*}}$, JINSHENG YU $^{1 *}$, GILBERTO SCHWARTSMANN $^{3}$, \\ HOWARD L. MCLEOD ${ }^{1}$ and JAMES W. FLESHMAN ${ }^{2}$
}

\begin{abstract}
Departments of ${ }^{1}$ Medicine and ${ }^{2}$ Surgery, Washington University School of Medicine and the Siteman Cancer Center, St. Louis, MO 63110, USA; ${ }^{3}$ Department of Gastroenterology, Post-Graduation in Science of Medicine, Universidade Federal do Rio, Grande do Sul, Brazil
\end{abstract}

Received October 11, 2010; Accepted December 21, 2010

DOI: $10.3892 /$ or.2011.1208

\begin{abstract}
Colorectal cancer is an endemic disease in the Western world. Search for molecular signatures present in primary tumors that predict tumor metastasis potential has been proposed and in particular, a 17-gene molecular signature is associated with poor survival in breast cancer, prostate cancer, meduloblastoma and lymphoma in a recent study. Using quantitative real-time PCR assay (qPCR), our study observed tumor-normal differential RNA expression in 15 of these 17 genes in a cohort of 52 stage III colorectal cancer patients (all $\mathrm{P}<0.05$ ), which signified the importance of these 17 signature genes in colorectal cancer. Although no significant correlation was found between tumor RNA levels of these 17 genes and some of clinical features (age, gender, and location, all $\mathrm{P}>0.05$ ), two distinct groups among these genes were observed with Spearman correlation scores $>0.6$ $(\mathrm{P}<0.01)$, suggesting co-expression/interaction within these genes. Of the 37 patients for whom complete follow-up data was available, 12 patients had recurrence and 25 had no recurrence. There was no significant difference in tumor RNA levels between recurrence and non-recurrence groups for the 17 genes (all $\mathrm{P}>0.05$ ), but the recurrence group had more patients with mucinous tumors $(9 / 12$ vs. $7 / 25, \mathrm{P}<0.05)$ and more lymph node involvement (median 7.2 vs. 2.5, $\mathrm{P}<0.05$ ) compared to the non-recurrence group. Moreover, survival analysis revealed a significant difference in patient overall survival time between low and high tumor RNA levels for 1 of the 17 genes (PTTG1, P=0.024). Our qPCR validation study confirms the importance of most 17-gene molecular
\end{abstract}

Correspondence to: Dr James W. Fleshman, Department of Surgery, Washington University School of Medicine, Campus Box 8109, 660 S. Euclid Ave, St. Louis, MO 63110, USA E-mail: fleshmanj@wustl.edu

*Contributed equally

Key words: gene expression, metastasis, colorectal cancer, quantitative PCR signature genes with differential RNA expression and suggests the relevance of PTTG1 for survival in colorectal cancers.

\section{Introduction}

Colorectal cancer (CRC) is an endemic disease in the Western world and it is the second most common cause of death for both men and women in the United States (1). Even though there are major advances in diagnosis and treatment of the disease, the mortality remains almost unaltered in the last 20 years (2). Initial CRC treatment is surgical, where staging is the determinant factor in prognosis and therapeutic decisions. Approximately $50 \%$ of patients who have curative surgical resection will die of a disease secondary to metastasis after surgery, and $80 \%$ of those will have recurrence of the disease detectable within 2 years (1-3).

The advancement of DNA manipulation techniques have promoted a significant increase in the knowledge of molecular mechanisms involved in the development and progression of cancer. Genomic analyses have supplied new markers that can definitely help in prognostic and therapeutic prediction based on tumor biology at molecular level. However, several promising colorectal cancer progression markers, such as p53, K-Ras, and cytokeratins, have not shown uniform results (4-7). Many of these studies were based on a small, nonconsecutive series of patients, and the patient selection was different in many ways such as variation in treatment time, different mutations of tumors, research methods, all making comparisons more difficult.

The studies have begun to identify groups of genes, the molecular signatures, whose alterations are associated with tissue abnormalities or altered outcome $(8,9)$. This has allowed description of new sub-classifications of patients based on genomic analysis, which is different from traditionally clinical-pathological analysis (10). Reports of successful applications in hematological malignancies, breast cancer $(11,12)$, bladder cancer $(13)$, malignant melanomas (14) have recently shown the significance of molecular signatures in primary tumors as prognostic markers based upon the characteristics of gene expression that can predict malignant potential of the tumors. In the studies of CRC, 
some new genes in the carcinogenous process have been described that can be used to determine new sub-classifications for staging based on the genomic analyses, and such molecular approaches are much more promising than the clinicalpathological parameters alone (15). For instance, Wang and colleagues using microarray technology have identified a 23gene signature that predicts recurrence in Dukes' B patients with $78 \%$ overall accuracy (16). The same approach has been utilized in the attempt of determining the differences of the gene expression between the tumors and the normal colonic tissue (17).

Metastasis is the main event that leads to death of a cancer patient. The search for molecular signatures present in a primary tumor that can identify the metastasis potential of each tumor has been described with promising results. Rasmaswamy has proposed a 17-gene molecular signature for metastasis in solid tumors (18). By using microarray technology in Rasmaswamy's study, primary adenocarcinomas from breast, prostate, lung, colon, uterus and ovary are compared with the metastasis from these adenocarcinomas. Through refining and gene clustering, a group of 17 genes that showed the molecular signature of metastasis in primary adenocarcinomas has been identified (Table I). The solid tumors presenting with the molecular signature of metastasis have a high tumor recurrence and consequently a worst clinical outcome. This 17-gene signature has also demonstrated association with survival rates in breast cancer, prostate cancer, meduloblastoma, and lymphoma. This model of molecular signature supports the hypothesis that primary tumors are pre-configured to metastasize and that this propensity can be detected in the primary tumor at the initial diagnosis.

Using quantitative real-time PCR in our study, we have analyzed these 17 genes in human colorectal cancer and paired normal colon tissues in order to further assess the significance of this model of molecular signature in predicting outcome of patients with colorectal cancer.

\section{Materials and methods}

Patients and samples. Tumor specimens and paired normal colon tissues used in this study were from 52 stage III colorectal cancer patients [29 male/23 female; age range, 39-96 (median, 79)]. Samples were snap-frozen in liquid nitrogen immediately after surgery and stored at $-80^{\circ} \mathrm{C}$. None of the patients had received preoperative radiation or chemotherapy. Tumor classification and differential grade were evaluated in all of the cases: 41 enteric adenocarcinoma, and 11 mucinous, and 1 grade I, 38 grade II, 13 grade III. Twenty-eight tumors were localized in the right colon, 18 in the left colon, and the remaining 6 in the rectum. The complete data from recurrence was collected in 37 patients, 25 had no recurrence (67.6\%), and 12 with recurrence $(32.4 \%)$. All recurrences were proved by biopsies or imaging studies, and were considered distant metastasis. The range of follow-up was a median of 19.9 months with limited available data. Written informed consent was obtained from all of the patients for the investigators to bank tumor tissue and to perform genomic analysis. This study was approved by the Washington University Human Subjects Committee.

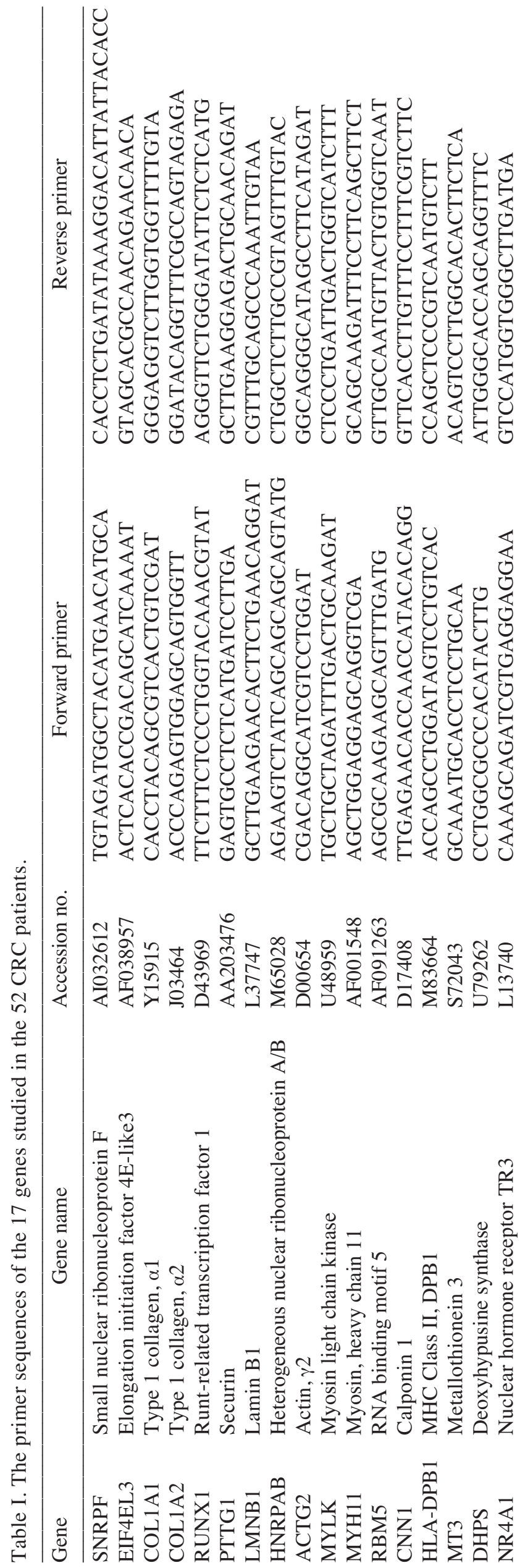


Genes studied. The 17 genes which are from the previous study (18) were chosen to be studied (Table I). Primers of these 17 genes were designed using the software Primer Express 2.0 (ABI, Foster City, CA) and sequence information for these primers was displayed in Table I. All primers were tested, the optimal annealing temperature was determined with gradient PCR for all 17 genes, and a single band at the right position with relevant amplicon size was confirmed with gel visualization. In addition, the dissociation curve feature in the ABI 7900 system showed the specific amplification for all 17 genes with a single peak of the melting temperature.

Quantitative real-time reverse transcription-PCR. Regions of high tumor cellularity were selected for RNA extraction (median $86.3 \%$; range, 65-95\%). Tissue total RNA was isolated from the colon tumor or adjacent normal colon mucosa with the TRIzol RNA isolation kit (Invitrogen, Carlsbad, CA), and the quality of RNA (i.e. A260/280>1.8 and the clear RNA bands for $28 \mathrm{~S}, 18 \mathrm{~S}$ and $5 \mathrm{~S}$ were seen) was confirmed in the Siteman Cancer Center Tissue Procurement Core. After reverse-transcription into cDNA using Superscript II reverse transcriptase (Invitrogen), the RNAs of 17 genes were determined by real-time qPCR, which was carried out in a $10-\mu 1$ reaction mix containing $2 \mu 1$ of cDNA $(10 \mathrm{ng} / \mu \mathrm{l})$, $5 \mu 1$ of 2 X SYBR-Green universal qPCR master mix (ABI), and $3 \mu \mathrm{l}$ or primer mix ( $400 \mathrm{nM}$ each forward and reverse primers). All real-time qPCR assays were performed in triplicate on an ABI PRIMSM 7900 Sequence Detector System (ABI) with the following program: $50^{\circ} \mathrm{C}$ for $2 \mathrm{~min}$ to activate uracil $\mathrm{N}$-glycosylase enzyme, and 40 cycles at $95^{\circ} \mathrm{C}$ for $15 \mathrm{sec}, 54$ or $6^{\circ} \mathrm{C}$ for $30 \mathrm{sec}$, and $72^{\circ} \mathrm{C}$ for $30 \mathrm{sec}$. The sequence detection program calculates a threshold cycle number (CT) at which the reporter fluorescence generated by cleavage of the probe is statistically greater than that of the background signal.

Measurement of relative RNA expression level. In this study, relative expression level was calculated using a modified comparative $\mathrm{C}_{\mathrm{T}}$ method, which uses actual real-time qPCR amplification efficiency instead of assuming all sets of gene primer and probe have approximately equal efficiency (19). The gene encoding amyloid B precursor protein was used as an internal reference gene, because it had near identical expression between colon tumor and normal tissues in previous serial analysis of gene expression analysis and less than a 3 -fold change between all tumor and normal sample pairs in this study. The relative expression level of an individual target gene was normalized to the reference gene and to one of all 104 colon tumor and normal RNA samples that was with the maximum CT value (i.e., the lowest expression level, called calibrator sample or $1 \mathrm{X}$ sample) in any target gene. A mathematical model was applied to determine the normalized relative expression level of a target gene in the individual sample, using the following formula:

$$
\text { Expression }=\frac{\mathrm{E}_{\text {target }}^{\Delta \mathrm{C}_{\mathrm{T}} \text { (calibrator-sample) }}}{\mathrm{E}_{\text {reference }}^{\left.\Delta \mathrm{C}_{\mathrm{T}} \text { (calibrator-reference }\right)}}
$$

where $\mathrm{E}$ target is the real-time qPCR efficiency of target gene transcript, and E reference is the real-time qPCR efficiency of reference gene transcript (20). Therefore, relative RNA expression level of a gene in this study is a relative number based on the reference gene and the $1 \mathrm{X}$ sample.

Statistical analysis. Statistical analyses were performed with the software STATISTICA (StatSoft, Inc., Tulsa, OK). Significance of the difference of relative expression level between paired tumor and normal samples was evaluated by Wilcoxon matched-pairs test. The influence of gender, tumor location, pathological variables was evaluated with MannWhitney or Kruskal-Wallis test. Correlation between variables was observed with Spearman rank correlation. Survival analysis was carried out with the SAS LIFETEST procedure (Cary, NC). The significance level was set at $\mathrm{P}<0.05$. P-values from multiple tests were corrected with the Bonferroni test.

\section{Results}

RNA expression of the 17 genes in colorectal tumor and normal tissues. Fifty-two samples from colorectal tumors and normal tissues each were studied simultaneously. There was a significant difference in RNA expression level between tumor and normal tissues in 15 of the 17 genes analyzed $(\mathrm{P}<0.05$ all), and the RNA expression of the remaining 2 genes (DHPS and NR4A1) had no significant difference between tumor and normal tissues (Fig. 1).

Ratio expression of the 17 genes between tumor and normal samples. The 17 genes were classified according to the ratio of RNA expression between tumor and normal tissues; and the tumor-normal ratio of the RNA expression was considered up-regulated when the ratio was $>1.2$, and down-regulated when $<0.8$. Of the 17 genes, 7 genes were classified as upregulated (SNRPF, EIF4EL3, COL1A1, COL1A2, PTTG1, RUNX1, LMNB1; P<0.05), another 7 were down-regulated (ACTG2, MYLK, MYH11, RBM5, CNN1, HLA-DPB1, MT3; $\mathrm{P}<0.05)$, and the rest 3 genes statistically had no difference in RNA expression between tumor and normal tissue as shown in Table II.

Co-expression of the 17 metastasis genes. Correlations of the RNA expression between all 17 genes were determined in 52 colorectal tumors. Statistically significant correlations were demonstrated in 2 distinct gene-groups. The first group had 4 genes with up-regulated and 2 with normal RNA expression (all Spearman coefficient $>0.6$, Table III). The other group was formed with 10 genes, of which 7 were down-regulated, 2 up-regulated, and 1 had normal RNA expression (all Spearman coefficient $>0.6$, Table III). The gene MT3 was not shown to have any strong correlation with the other 16 genes. No evidence of such co-expression was observed in the normal tissues.

Tumor RNA expression and patient clinical features. The comparisons of the RNA expression were made with patient age, gender, tumor location, histological type, pathological grade, lymph node involvement, and recurrence at the last follow-up. There was no statistically significant difference in 


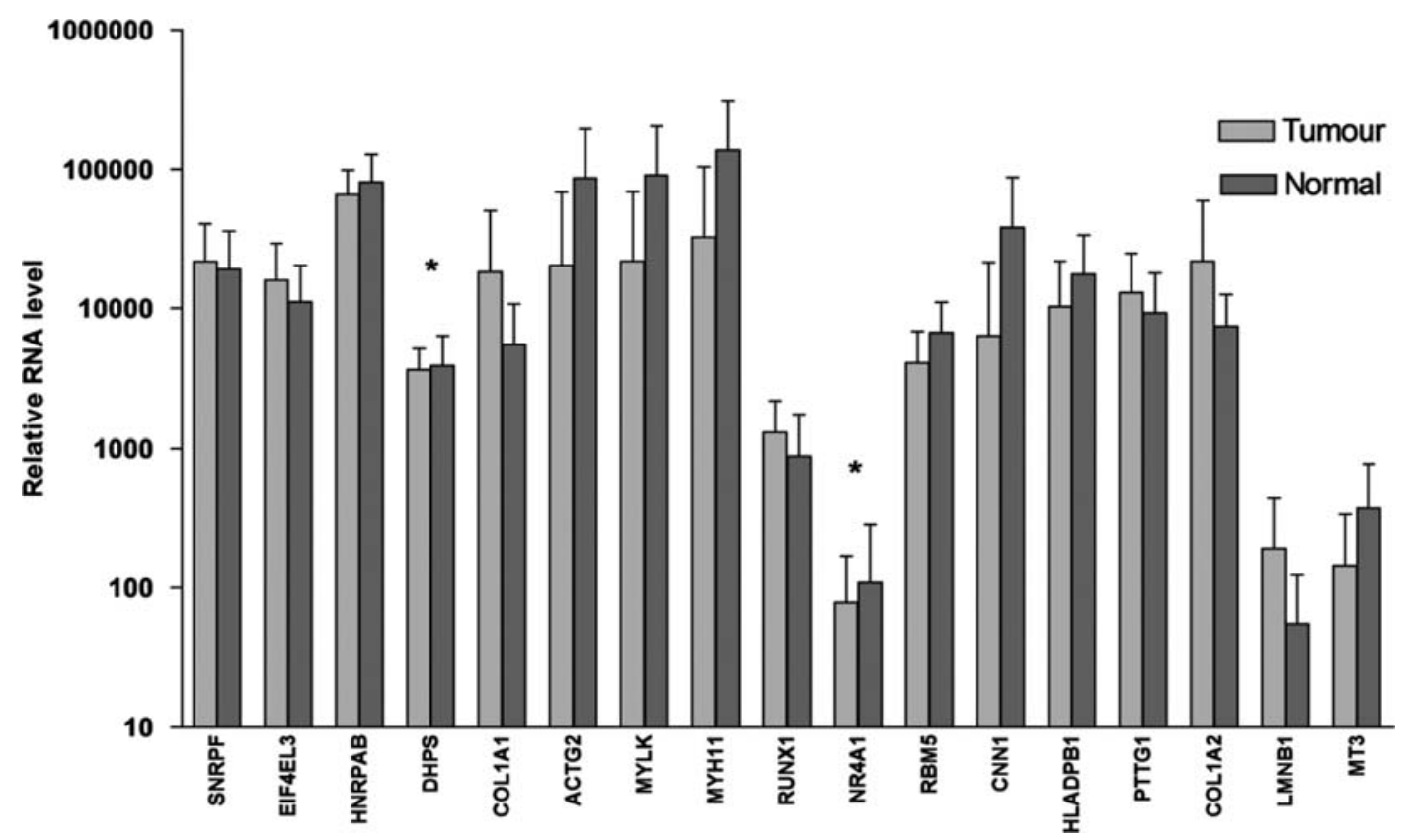

Figure 1. Differential expression in relative RNA level of the 17 genes between tumor and matched normal mucosa tissues in 52 colorectal cancer patients. $\left({ }^{*} \mathrm{P}>0.05\right.$ for DHPS and NR4A1, $\mathrm{P}<0.05$ in the rest 15 genes). Error bars represent $95 \% \mathrm{CI}$ of the mean. All assay values were normalized to the internal reference gene APP.

Table II. T/N category of the RNA expression in 52 CRC patients.

\begin{tabular}{lcccc}
\hline Gene symbol & $\begin{array}{c}\text { No. of cases in category } \\
\text { of } \mathrm{T} / \mathrm{N}<0.8\end{array}$ & $\begin{array}{c}\text { No. of cases in category } \\
\text { of } \mathrm{T} / \mathrm{N}=0.8-1.2\end{array}$ & $\begin{array}{c}\text { No. of cases in category } \\
\text { of T/N }>1.2\end{array}$ & P-value \\
\hline Up-regulated & & & & 0.023 \\
SNRPF & 13 & 11 & 24 & 0.001 \\
EIF4EL3 & 12 & 7 & 29 & 0.000 \\
COL1A1 & 11 & 5 & 32 & 0.000 \\
COL1A2 & 12 & 4 & 32 & 0.000 \\
RUNX1 & 12 & 5 & 31 & 0.000 \\
PTTG1 & 14 & 6 & 28 & 0.000 \\
LMNB1 & 7 & & 25 & 0.000 \\
Down-regulated & & 2 & & 0.000 \\
ACTG2 & 34 & 5 & 12 & 0.000 \\
MYLK & 36 & 5 & 7 & 0.000 \\
MYH11 & 36 & 14 & 7 & 0.000 \\
RBM5 & 29 & 2 & 10 & 0.000 \\
CNN1 & 36 & 6 & 8 & 0.000 \\
HLA-DPB1 & 34 & 6 & 11 & \\
MT3 & 31 & & & 0.134 \\
Normal & & 11 & 15 & 1.000 \\
HNRPAB & 22 & 12 & 18 & 0.845 \\
DHPS & 18 & 3 & 23 & \\
NR4A1 & 22 & & & \\
\hline
\end{tabular}

tumor RNA expression of these 17 genes across all different clinical-pathological features when analyzed as a single marker (all $\mathrm{P}>0.05$ ). However, two groups of patients were separated using the end point of clinical recurrence, and 37 patients of these two groups had complete clinical data available for further analysis. Of the 37 patients, 12 patients had recurrence and 25 had no recurrence. Although there was statistically no significant difference in RNA expression of the 17 genes between these 2 groups $(\mathrm{P}>0.05)$, but several pathological features such as tumor histological type and 


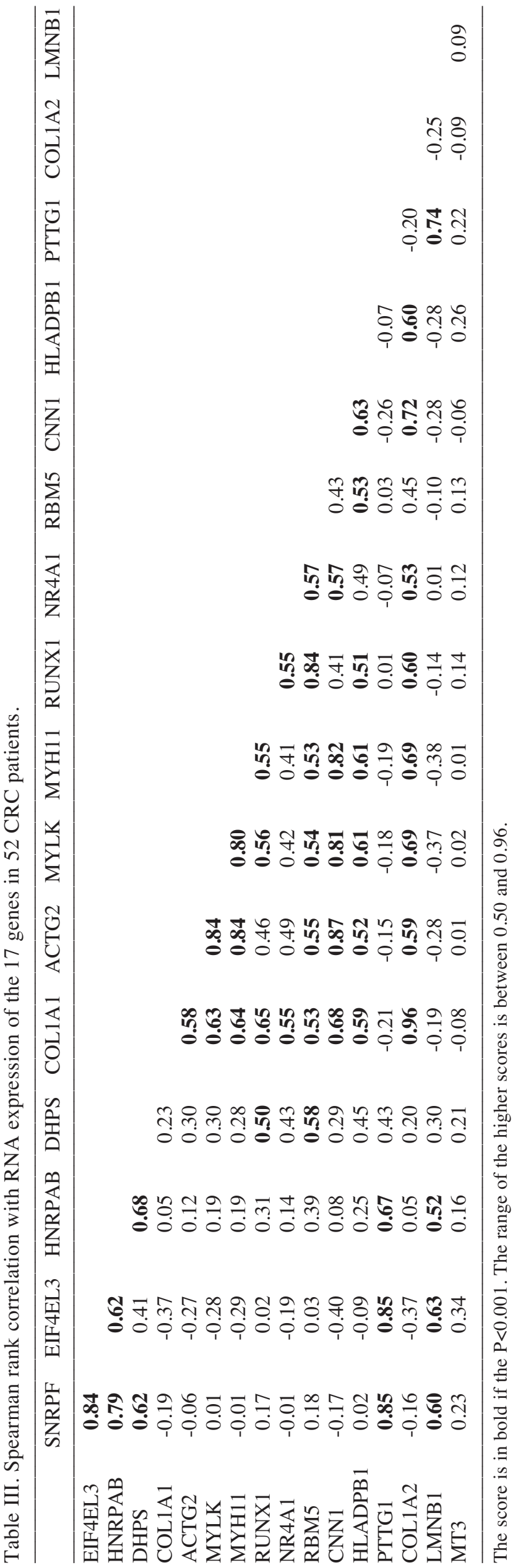

lymph node involvement were statistically different between the recurrence and non-recurrence groups $(\mathrm{P}=0.02$ and 0.01 , respectively), i.e., the recurrence group had more patients with mucinous tumor than the non-recurrence group (9/12 vs. $7 / 25, \mathrm{P}<0.05)$ and there was a 7.2 vs. 2.5 of median lymph node involvement for the recurrence and non-recurrence groups $(\mathrm{P}<0.05)$, respectively. Moreover, survival analysis revealed a significant difference in patient overall survival time between low and high tumor RNA levels for 1 of the 17 genes (PTTG1, P=0.024) (Fig. 2).

Hierarchical clustering analysis in 52 colorectal tumors. Clustering analysis with tumor RNA expression of the 17 genes was performed in the 52 patients with colorectal cancer; and 3 distinct patient-groups were found (Fig. 3). The tumor RNA expression was statistically significantly different between these 3 groups $(\mathrm{P}<0.05)$ in majority of the 17 genes (14/17), but not in the other 3 genes (MT3, DPHS, and HNRPAB). No significant difference was found for each of the clinical-pathological features between these 3 patientgroups separated by clustering with tumor RNA expression $(\mathrm{P}>0.05)$. These correlations were tested with patient age, gender, tumor location, histological type, pathological grade, differentiation, invasion, lymph node involvement, and status of disease and recurrence. However, the hierarchical clustering analysis had not shown these 3 to be distinct or any patientgroups in 52 normal tissues.

\section{Discussion}

The 17-gene signature that was previously related to poor prognosis in different solid tumors (18) has been evaluated in our study for the CRC stage III patients. Our study demonstrated that the RNA expression in CRC tumors is significantly different from matched normal mucosa, independent of anatomic and clinical pathological variables in 15 out of 17 genes $(\mathrm{P}>0.05)$. These results might be a confirmation that this group of genes can be involved in the development of colorectal cancer, and probably in other solid tumors as well. The differential expression between tumor and normal tissues from CRC patients also suggests that these 17 genes may have important role in tumor biology which favors tumor classification and decision making for optimized therapy. However, the RNA expressions of these 17 genes in the tumors were not associated with disease recurrence or other clinical-pathological features in our study and rather the tumor type and lymph node involvement were shown to be useful for prediction of disease recurrence in the pre-therapy setting. These results did not establish a positive relationship between the 17-gene molecular signature and tumor recurrence/ metastasis. Nonetheless, one of the 17 genes (PTTG1) has been indicated a significant correlation with patient overall survival, which is a potential survival biomarker although more validation studies are needed. The difficulty to compare studies of molecular analysis in literature is mainly due to the techniques utilized and different selections of cancer patients. The techniques utilized should be validated carefully in such molecular analysis and results from such studies should be interpreted with caution. Michiels and colleagues (21) have re-analyzed the metadata from seven large studies 


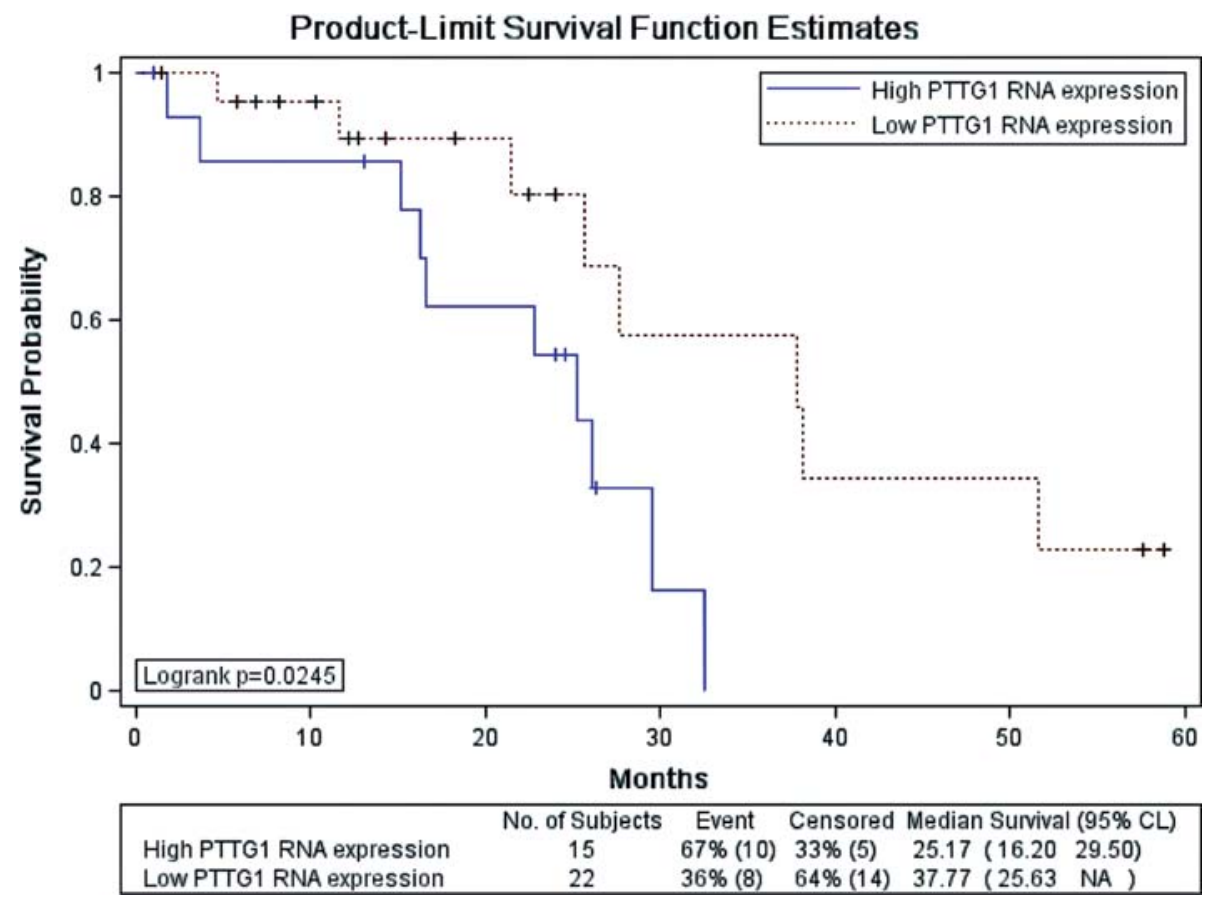

Figure 2. Survival curve for 37 colorectal cancer patients with follow-up data. The analysis was performed with the Kaplan-Meier estimate model in the SAS LIFETEST procedure.

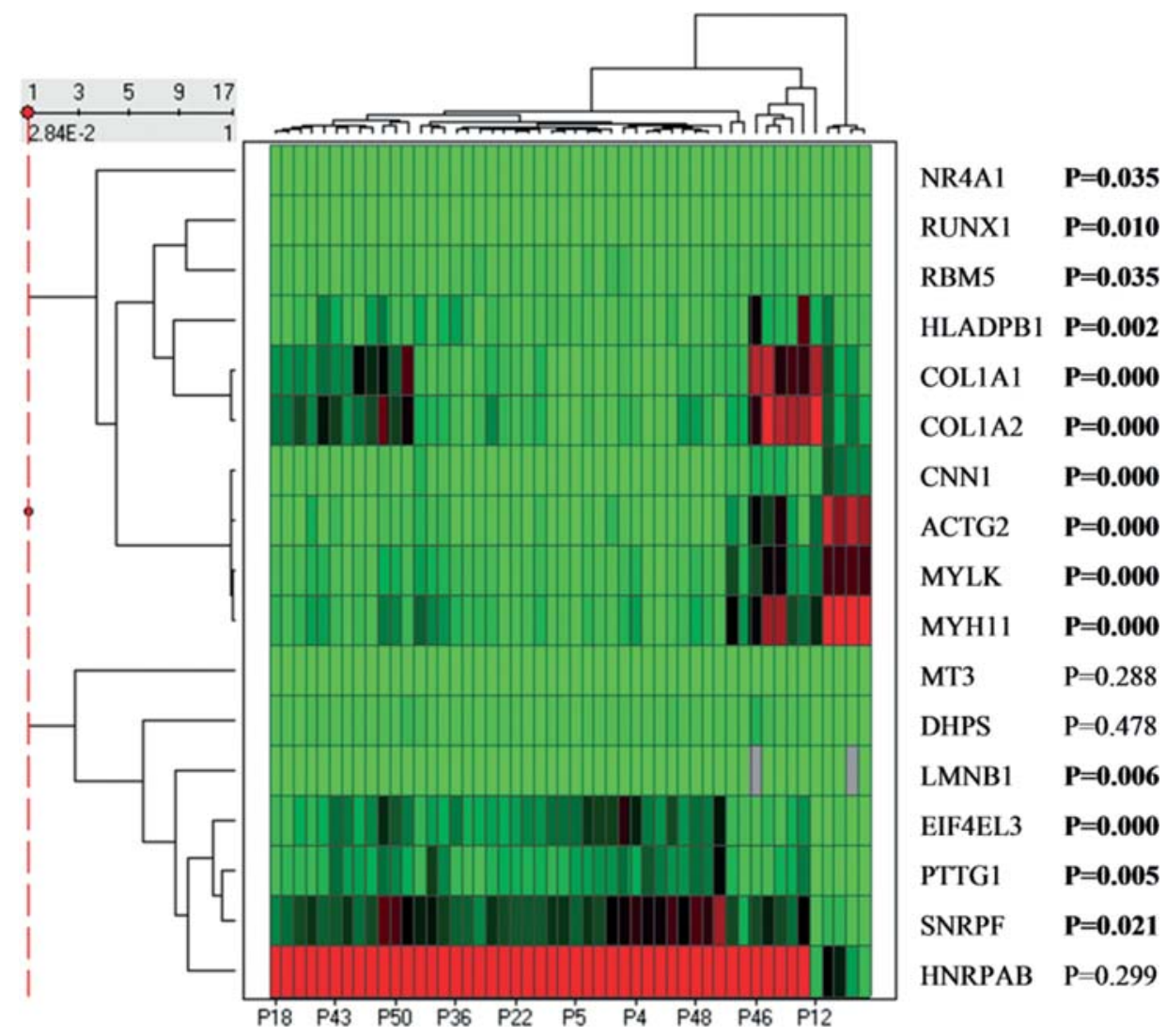

Figure 3. Hierarchical clustering heat map for tumor RNA expression in 52 CRC patients. Columns represent tumor samples and rows represent the 17 genes. Red and green represent relative high and low RNA expression levels, respectively.

that have attempted to predict prognosis of cancer patients on the basis of microarray analysis. The results from these metadata suggest that the list of genes identified as predictors of prognosis was highly inconsistent and molecular signatures strongly depended on the selection of patients in the training sets. For all but one study, the rate of misclassification 
decreased as the number of patients in the training set increased. Five of the seven studies did not classify patients better than chance. The conclusion is that the prognostic value of published microarray results in cancer studies should be considered with caution; and we advocate the use of validation by repeated random sampling (22).

Although no correlations between the tumor gene expression and clinical-pathological features were found in our study, hierarchic clustering based on the RNA expression in tumors has demonstrated the existence of 3 patient groups and all clinical-pathological parameters among those 3 groups were not significantly different, suggesting that the tumor RNA expression behaves in a different way on different groups of patients with the same stage. This might explain different biological behavior of tumors when classified by clinical-pathological parameters, even for patients in the same staging group. The characterization of molecular signatures among these groups may allow the determination of unique tumor biologic behaviors as well as different clinical evolution in the same group of patients, who are assigned into new different classifications on staging and may need different treatment options for each specific group.

Interactions between genes in a variety of cells are a major challenge for understanding of gene functions in complex cellular processes. How those genes work in a certain organ and how they interact are a complex process, which is the object of many studies. The big challenge will be to unveil how this interaction occurs among different genes biologically. In our study, there were two groups of genes that are positively correlated with each other in the RNA expression. The first group consisted of 10 genes and the other group of 6 genes. The normal tissue RNA expression of the 17 genes did not show such correlations in the groups. These findings suggest that there are interactions among those grouped genes, and require complementary studies to determine how these interactions occur.

In conclusion, our qPCR validation study confirms the importance of most 17-gene molecular signature genes with differential RNA expression, and suggests the survival relevance of PTTG1 in colorectal cancers. Additional studies should be done to define the real place and function of these 17 genes in colorectal tumors, their relationship with tumor metastasis, and the possible clinical applications.

\section{Acknowledgements}

This study was supported in part by the Siteman Cancer Center (P30 CA 091842), The BJH Foundation, the Pharmacogenetics Research Network (GM 63340; http://pharmacogenetics.wustl.edu), and the Section of Colorectal Surgery. The efforts of the members of the Siteman Cancer Center Tissue Procurement Core (Mark Watson) were greatly appreciated.

\section{References}

1. Jemal A, Siegel R, Ward E, Murray T, Xu J and Thun MJ: Cancer statistics, 2007. CA Cancer J Clin 57: 43-66, 2007.

2. Smith RA, Cokkinides V and Eyre HJ: Cancer screening in the United States, 2007: a review of current guidelines, practices, and prospects. CA Cancer J Clin 57: 90-104, 2007.

3. Niu R, Yan TD, Zhu JC, Black D, Chu F and Morris DL: Recurrence and survival outcomes after hepatic resection with or without cryotherapy for liver metastases from colorectal carcinoma. Ann Surg Oncol 14: 2078-2087, 2007.

4. Wong SC, Chan CM, Ma BB, et al: Clinical significance of cytokeratin 20-positive circulating tumor cells detected by a refined immunomagnetic enrichment assay in colorectal cancer patients. Clin Cancer Res 15: 1005-1012, 2009.

5. Watanabe T, Wu T and Catalano P: Molecular predictors of survival after adjuvant chemotherapy for colon cancer. $\mathrm{N}$ Engl J Med 344: 1196-1206, 2001.

6. Calistri D, Rengucci C, Seymour I, et al: KRAS, p53 and BRAF gene mutations and aneuploidy in sporadic colorectal cancer progression. Cell Oncol 28: 161-166, 2006.

7. Walther A, Johnstone E, Swanton C, Midgley R, Tomlinson I and Kerr D: Genetic prognostic and predictive markers in colorectal cancer. Nat Rev Cancer 9: 489-499, 2009.

8. Khan A: Genomics and microarray for detection and diagnostics. Acta Microbiol Immunol Hung 51: 463-467, 2004.

9. Hegde P, Qi R, Gaspard R, et al: Identification of tumor markers in models of human colorectal cancer using a 19.200element complementary DNA microarray. Cancer Res 61: 7792-7797, 2001.

10. Alizadeh A, Eisen M, Davis R, et al: Distinct types of diffuse large B-cell lymphoma identified by gene expression profiling. Nature 403: 503-511, 2000

11. Sorlie T, Perou C, Tibshirani R, et al: Gene expression patterns of breast carcinomas distinguish tumor subclasses with clinical implications. Proc Natl Acad Sci 98: 10869-10874, 2001

12. van de Vijver M, He Y, van't Veer L, et al: A gene-expression signature as a predictor of survival in breast cancer. N Engl J Med 347: 1999-2009, 2002.

13. Thykjaer T, Workman C, Kruhoffer M, et al: Identification of gene expression patterns in superficial and invasive human bladder cancer. Cancer Res 61: 2492-2499, 2001.

14. Jaeger J, Koczan D, Thiesen HJ, et al: Gene expression signatures for tumor progression, tumor subtype, and tumor thickness in laser-microdissected melanoma tissues. Clin Cancer Res 13: 806-815, 2007.

15. Frederiksen C, Knudsen S, Laurberg S and Orntoft T: Classification of Dukes' B and C colorectal cancers using expression arrays. J Cancer Res Clin Oncol 129: 263-271, 2003.

16. Wang Y, Jatkoe T, Zhang Y, et al: Gene expression profiles and molecular markers to predict recurrence of Dukes' B colon cancer. J Clin Oncol 22: 1564-1571, 2004.

17. Yu J, Shannon WD, Watson MA and McLeod HL: Gene expression profiling of the irinotecan pathway in colorectal cancer. Clin Cancer Res 11: 2053-2062, 2005.

18. Ramaswamy S: A molecular signature of metastasis in primary solid tumors. Nat Genet 33: 49-54, 2003.

19. Yu J, Mallon MA, Zhang W, et al: DNA repair pathway profiling and microsatellite instability in colorectal cancer. Clin Cancer Res 12: 5104-5111, 2006.

20. Pfaffl M: A new mathematical model for relative quantification in real-time RT-PCR. Nucleic Acids Res 29: e45, 2001.

21. Michiels S, Koscielny S and Hill C: Prediction of cancer outcome with microarrays: a multiple random validation strategy. Lancet 365: 488-492, 2005.

22. Fritzmann J, Morkel M, Besser D, et al: A colorectal cancer expression profile that includes transforming growth factor beta inhibitor BAMBI predicts metastatic potential. Gastroenterology 137: 165-175, 2009. 